\title{
BMJ Open Diagnostic and Prognostic Biomarkers in the Rational Assessment of Mesothelioma (DIAPHRAGM) study: protocol of a prospective, multicentre, observational study
}

Selina Tsim, ${ }^{1,2}$ Caroline Kelly, ${ }^{3}$ Laura Alexander, ${ }^{3}$ Carol McCormick, ${ }^{3}$ Fiona Thomson, ${ }^{3}$ Rosie Woodward, ${ }^{4}$ John E Foster, ${ }^{4}$ David B Stobo, ${ }^{5}$ Jim Paul, ${ }^{3}$ Nick A Maskell, ${ }^{6}$ Anthony Chalmers, ${ }^{2,7}$ Kevin G Blyth ${ }^{1,8}$

To cite: Tsim S, Kelly C, Alexander L, et al. Diagnostic and Prognostic Biomarkers in the Rational Assessment of Mesothelioma (DIAPHRAGM) study: protocol of a prospective, multicentre, observational study. BMJ Open 2016;6:e013324. doi:10.1136/bmjopen-2016013324

- Prepublication history and additional material is available. To view please visit the journal (http://dx.doi.org/ 10.1136/bmjopen-2016013324)

Received 4 July 2016 Revised 24 October 2016 Accepted 25 October 2016

CrossMark

For numbered affiliations see end of article.

Correspondence to Dr Kevin G Blyth; kevin.blyth@ggc.scot.nhs.uk

\section{ABSTRACT}

Introduction: Malignant pleural mesothelioma (MPM) is an asbestos-related cancer, which is difficult to diagnose. Thoracoscopy is frequently required but is not widely available. An accurate, non-invasive diagnostic biomarker would allow early specialist referral, limit diagnostic delays and maximise clinical trial access. Current markers offer insufficient sensitivity and are not routinely used. The SOMAmer proteomic classifier and fibulin-3 have recently demonstrated sensitivity and specificity exceeding $90 \%$ in retrospective studies. DIAPHRAGM (Diagnostic and Prognostic Biomarkers in the Rational Assessment of Mesothelioma) is a suitably powered, multicentre, prospective observational study designed to determine whether these markers provide clinically useful diagnostic and prognostic information.

Methods and analysis: Serum and plasma (for SOMAscan and fibulin-3, respectively) will be collected at presentation, prior to pleural biopsy/pleurodesis, from 83 to 120 patients with MPM, at least 480 patients with non-MPM pleural disease and 109 asbestos-exposed controls. Final numbers of MPM/ non-MPM cases will depend on the incidence of MPM in the study population (estimated at 13-20\%). Identical sampling and storage protocols will be used in 22 recruiting centres and histological confirmation sought in all cases. Markers will be measured using the SOMAscan proteomic assay (SomaLogic) and a commercially available fibulin-3 ELISA (USCN Life Science). The SE in the estimated sensitivity and specificity will be $<5 \%$ for each marker and their performance will be compared with serum mesothelin. Blood levels will be compared with paired pleural fluid levels and MPM tumour volume (using MRI) in a nested substudy. The prognostic value of each marker will be assessed and a large bioresource created.

Ethics and dissemination: The study has been approved by the West of Scotland Research Ethics Committee (Ref: 13/WS/0240). A Trial Management Group meets on a monthly basis. Results will be published in peer-reviewed journals, presented

\section{Strengths and limitations of this study}

Prospective, multicentre study recruiting a representative sample of patients in an intention-to-diagnose population.

- Strict sampling, processing and storage methods used in all patients.

- Robust diagnostics and 12 months' follow-up

- Creation of a large bioresource annotated with detailed, prospectively collected clinical information, for use in future biomarker discovery and validation studies.

- The final number of study participants with malignant pleural mesothelioma, and therefore the power available to test the primary objective, will not be known until recruitment is complete.

at international meetings and disseminated to patient groups.

Trial registration number: ISRCTN10079972, Pre-results.

\section{INTRODUCTION}

Malignant pleural mesothelioma (MPM) is an invasive thoracic malignancy, strongly associated with prior asbestos exposure. The median survival for patients with MPM is poor at 9-10 months. ${ }^{12}$ However, the prognosis of individuals is highly variable and largely determined by histological subtype. ${ }^{2}$ MPM frequently presents as an emergency with a large, symptomatic pleural effusion. ${ }^{3}$ Early specialist referral is frequently required because pleural fluid aspiration cytology is unreliable $^{4}$ and histological confirmation is recommended in all patients. ${ }^{5}$ Thoracoscopy (under local or general anaesthesia) ${ }^{5}$ enables widespread tissue sampling $^{6}$ with 
diagnostic yields for malignancy $>90 \%{ }^{7}$ but is not available in all centres. Thoracoscopy also allows pleurodesis or indwelling pleural catheter placement.

A reliable, non-invasive diagnostic biomarker for MPM would be a major clinical advance. This would allow clinicians to reliably differentiate likely MPM from secondary pleural malignancies (eg, lung or breast cancer), which may present with similar clinical and imaging features but require less evolved diagnostic pathways. This reflects the improved sensitivity of pleural cytology in these diseases ${ }^{8-10}$ and the frequent option of alternative sites for tissue biopsy. A positive MPM biomarker test could facilitate early referral to a thoracoscopy centre and avoid unnecessary diagnostic delay (eg, due to repeated pleural aspirations), minimising the risk of subsequent needle-tract metastases ${ }^{11}{ }^{12}$ and maximising opportunity for clinical trial enrolment. Previous studies have demonstrated that blood levels of single proteins, including mesothelin, ${ }^{13}{ }^{14}$ megakaryocyte potentiating factor (MPF) ${ }^{15}$ and osteopontin, ${ }^{16}$ are higher in patients with MPM than in asbestos-exposed controls (AECs) and patients with secondary pleural malignancies. Mesothelin, a cell-adhesion glycoprotein that is overexpressed in MPM ${ }^{17} 18$ is the most widely studied and is associated with an MPM sensitivity of $56-77 \%$ at $95 \%$ specificity $^{14} 15{ }^{19}$ but much reduced performance in patients with non-epithelioid MPM. A recent meta-analysis (of 4491 individuals (1026 with MPM)) reported a sensitivity of only $32 \%$ at $95 \%$ specificity. Mesothelin does not, therefore, contribute to current diagnostic algorithms. ${ }^{20}$ MPF offers no advantage over mesothelin, ${ }^{15}$ while the clinical utility of osteopontin is limited by stability and reproducibility concerns. ${ }^{16}$

An ideal MPM biomarker would be measurable in blood for ease of collection and offer sufficient sensitivity at high specificity in patients presenting with suspected MPM. Differentiation between advanced disease patients and appropriate controls is of limited value. High specificity is mandatory for a low prevalence disease, and should apply to patients with asbestos exposure and non-MPM pleural disease. Biomarker results should also correlate with disease extent and have defined relationships with potential confounders including renal function $^{21}$ and the effect of pleural interventions. The latter is important because the precedent has been established in prostate ${ }^{22} 23$ and breast cancer ${ }^{24}$ that recent sampling, resection or peritumoural inflammation may affect biomarker expression. This is particularly relevant to MPM where biopsies are frequently large and often combined with pleurodesis. Several previous biomarker studies, which validated inconsistently in external populations, used samples acquired at later time points, often postdiagnosis (and postpleurodesis) including samples taken prior to, during or after resection surgery. ${ }^{162526}$ The aim of the DIAPHRAGM (Diagnostic and Prognostic Biomarkers in the Rational Assessment of Mesothelioma) study is to prospectively evaluate the diagnostic and prognostic performance of the SOMAscan proteomic classifier $^{27}$ and fibulin- $3,{ }^{25}$ which have demonstrated high sensitivity and specificity in recent retrospective series. The study has been designed to generate clinically meaningful results, which can be related to MPM biology and confounding factors, and applied to patients at first presentation.

\section{SOMAmer-based proteomic classifier}

The SOMAscan assay is a highly multiplexed proteomic platform that uses SOMAmer (Slow Off-rate Modified Aptamers) reagents to selectively bind and quantify proteins. ${ }^{28}$ A 13-protein classifier was developed by SomaLogic (Boulder, Colorado, USA); using this novel proteomics-based biomarker detection technique ${ }^{27}$ in a retrospective study, over 800 proteins were measured in the serum of 117 patients with MPM and 142 AECs, collected at surgical MPM centres in the USA between 1996 and 2011. Using a panel of 13 differentially expressed proteins and a cut-point of 0.5 , the classifier was able to segregate MPM from controls with an area under the curve (AUC) of $0.99 \pm 0.01$ in training $(60 \mathrm{MPM} / 60$ controls), $0.98 \pm 0.04$ in blinded verification (19 MPM/20 controls) and $0.95 \pm 0.04$ in blinded validation sets (38 cases/62 controls). ${ }^{27}$ The combined sensitivity for the three cohorts was $93 \%$ at $91 \%$ specificity. Based on the published receiver operating characteristic (ROC) curve for the validation cohort, sensitivity at $95 \%$ specificity appeared to be $\sim 78 \%$, although the authors did not report this value. This performance exceeds that of any previous MPM biomarker, although the classifier's specificity appeared lower in patients with non-MPM pleural effusion $(n=32)$. There was a modest correlation between classifier score and disease stage, but prognostic significance was not assessed. The 13 classifier proteins (9 upregulated, 4 downregulated) have not previously been associated with MPM. Their functions fall into two broad groups: regulation of proliferation and inflammation. Quite apart from their biological relevance to MPM, the latter is an important potential confounder because many of the patients involved will have previously undergone pleurodesis. In addition, several groups have reported an independent interaction between prognosis and inflammatory biomarkers in MPM, including neutrophil-to-lymphocyte ratio, ${ }^{29-31}$ monocytosis ${ }^{32}$ and the modified Glasgow Prognostic Score. ${ }^{31}$ Therefore, adequate understanding of the diagnostic and prognostic utility of this assay requires replication in a prepleurodesis cohort and prospective evaluation of interactions between inflammatory biomarkers and SOMAscan scores.

\section{Fibulin-3}

Fibulin-3 is a secreted glycoprotein, encoded by the epidermal growth factor-containing fibulin-like extracellular matrix protein 1 gene. ${ }^{33}$ Fibulin-3 is overexpressed in MPM tumours relative to adjacent benign pleura ${ }^{25}$ and expressed and secreted by MPM cell lines. ${ }^{26}$ Pass $e t a l^{25}$ retrospectively measured fibulin-3 in the plasma of 
92 patients with MPM, 136 AECs, 93 patients with non-MPM pleural effusion and 43 healthy controls. A plasma cut-point of $52 \mathrm{ng} / \mathrm{mL}$ provided $97 \%$ sensitivity at $95 \%$ specificity and a $95 \%$ CI of the AUC of 0.97 to 0.99 in differentiating MPM from all other cases. However, in a blinded external validation set, sensitivity was below $40 \%$ (at $95 \%$ specificity), with an $\mathrm{AUC}=0.87$.

Subsequent studies have revealed mixed results. In a study of 153 patients (82 with MPM), Creaney et $a l^{14}$ reported a sensitivity of $22 \%$ (at $95 \%$ specificity) at the same $52 \mathrm{ng} / \mathrm{mL}$ cut-point and an AUC of 0.671 (0.606 to 0.732 ), which was significantly inferior to mesothelin measured in the same patients (sensitivity $56 \%$ (at $95 \%$ specificity); AUC 0.816 (0.755 to 0.867$)$ ) at a $2.5 \mathrm{nM}$ threshold). In a small Egyptian study using an unspecified fibulin-3 assay and internally defined cut-points, Agha $e t a l^{34}$ reported $100 \%$ sensitivity/78\% specificity in differentiating MPM cases $(n=25)$ from non-malignant pleural disease $(n=9)$, and $88 \%$ sensitivity/82\% specificity in differentiating MPM from secondary pleural malignancies $(\mathrm{n}=11)$. No combined sensitivity was reported. An Italian study found no difference in fibulin-3 levels but used serum (not plasma), a control group without pleural disease (asbestosis) and contained only 14 patients with MPM. ${ }^{35}$

\section{METHODS AND ANALYSIS \\ Study design}

DIAPHRAGM is a prospective, multicentre observational study. The study incorporates sampling windows that correspond to the proposed use of a diagnostic biomarker, that is, at presentation with suspected pleural malignancy (SPM). The overall study design is summarised in figure $1 \mathrm{~A}, \mathrm{~B}$. The main impact of this design is that biomarkers will be drawn before a diagnosis is made. In addition to better replicating the future use of these markers, this avoids the potential confounding effect of pleurodesis on biomarker results. The diagnostic performance of the SOMAmer panel and fibulin-3 will be assessed using cut-points determined in the relevant original studies and compared with mesothelin (using the MESOMARK ELISA (Fujirebio Diagnostics)). Identical processing and storage protocols will be used in patients with SPM and a group of AECs. Potential confounders including renal function, inflammatory indices and drugs will be recorded at all visits. The timing of the biomarker blood draw in relation to pleural aspiration (preaspiration or postaspiration) will be recorded in order to assess the effect of this intervention on biomarker results. An exploratory, cross-sectional MRI substudy will determine if there is any correlation between blood biomarker levels and MPM tumour volume, as has been established for mesothelin using CT-positron emission tomography scanning. ${ }^{36}$

\section{Study objectives and outcome measures}

These are presented in table 1 .

\section{Setting}

At least 600 consecutive patients with SPM will be recruited from 22 centres (20 in the UK, 1 in Republic of Ireland). These are a mixture of academic and more clinically orientated units. This should make the results of the DIAPHRAGM study generalisable to patients presenting with SPM to acute hospital services. The principal criterion used to select centres was that they had sufficiently evolved pleural diagnostic services to deliver a reliable diagnosis. Specifically, access to on-site thoracoscopy (ideally including local anaesthetic thoracoscopy (LAT)) and a regional mesothelioma multi-disciplinary team meeting (for diagnostic review and staging) was required.

\section{Screening and eligibility assessment \\ Suspected pleural malignancy}

Cases will be identified on presentation to a respiratory outpatient clinic or acute hospital admissions unit. This will be based on the history, examination and available investigations. Potentially eligible patients will be provided with the study patient information sheet (PIS, see online supplementary appendix 1) and eligibility assessed based on the following criteria:

Inclusion criteria:

- SPM, defined by a unilateral pleural effusion or pleural mass lesion;

- Sufficient fitness for diagnostic sampling (site investigator's clinical judgement);

- Informed written consent.

Exclusion criteria:

- Intercostal chest drain in situ, or inserted within the previous 3 months.

Asbestos-related pleural plaques are not an inclusion criterion since these are absent in up to $25 \%$ of MPM cases, ${ }^{37}$ and are also common in asbestos-exposed populations without MPM. ${ }^{38}$ Patients with lung nodules or other visceral mass lesions are not excluded, assuming the investigator suspects pleural malignancy. This is because of the high prevalence of lung nodules in the target population (older patients, commonly smokers) and the high false-positive rate of CT imaging in this regard. ${ }^{39}$

Participants recruited to the SPM arm will generate cohorts of MPM and non-MPM pleural disease of various aetiologies, likely including benign asbestosrelated pleural effusion and secondary pleural malignancies. These numbers will be sufficient to address the primary objective with sufficient statistical power to inform clinical practice (see later section).

\section{AEC participants}

One hundred and nine AECs will be recruited via invitations sent by Clydeside Action on Asbestos (CAA), an advocacy body based in Glasgow with a database of over 600 clients, or by respiratory clinics at the host centre. Individuals will be invited to participate by letter (if identified via CAA) or given the PIS (see online 
Figure 1 Summary of the design of the DIAPHRAGM study.

(A) Describes the optimal diagnostic pathway for the majority of patients who present with significant pleural effusion \pm pleural thickening or a pleural mass. (B) Describes the optimal diagnostic pathway for the minority of patients who present with an isolated pleural mass, but no significant fluid component. The pathway chosen is ultimately at the discretion of the investigating physician. DIAPHRAGM, Diagnostic and Prognostic Biomarkers in the Rational Assessment of Mesothelioma; MPM, malignant pleural mesothelioma.

\section{A}
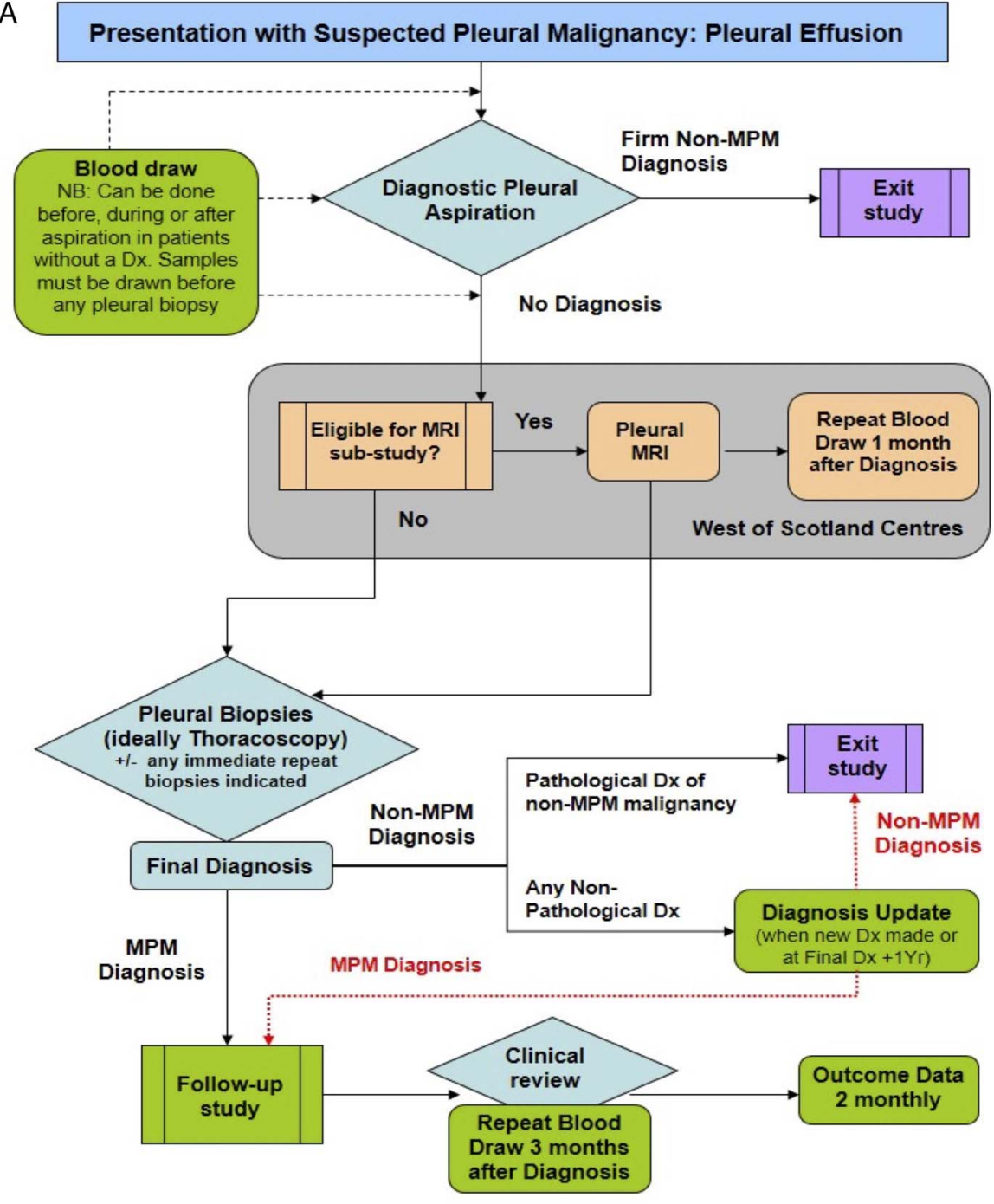

B

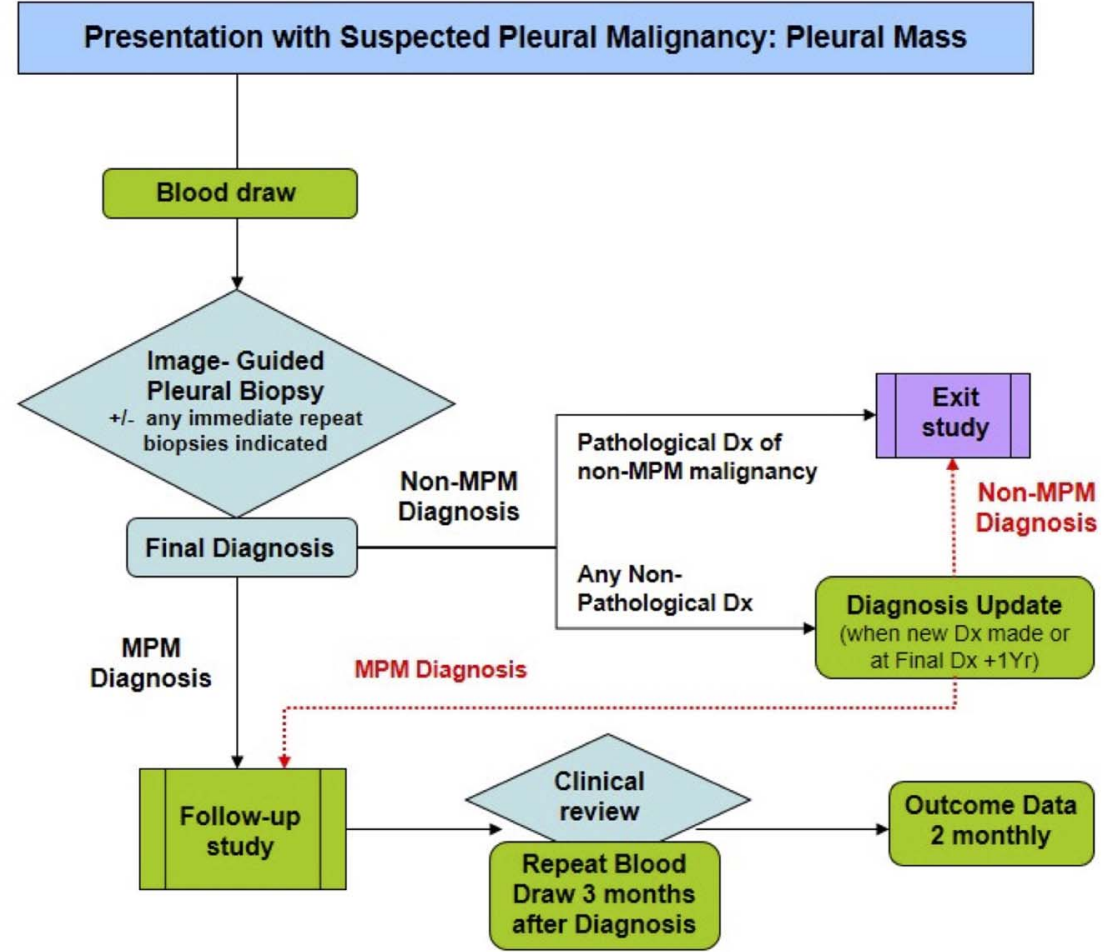


Table 1 Outcome measures used in the DIAPHRAGM study

\section{Research objective}

Primary

To determine whether SOMAscan results and/or fibulin-3 levels in blood at presentation can differentiate MPM from asbestos-exposed controls and patients with other causes of pleural effusion with a sufficient degree of sensitivity and specificity to be of routine clinical value

Secondary

To determine whether:

1. SOMAscan results and/or fibulin-3 levels at presentation provide clinically useful prognostic information in patients with MPM

2. Early changes in SOMAscan and/or fibulin-3 levels after diagnosis (at 3 months) are associated with a poorer prognosis in MPM

\section{Exploratory}

To determine whether:

1. There is a correlation between SOMAscan and/or fibulin-3 levels in blood and tumour volume, defined by MRI

2. There is a correlation between SOMAscan and/or fibulin-3 levels in blood and tumour angiogenesis (as defined by perfusion-based MRI biomarkers)

3. There is a correlation between SOMAscan and/or fibulin-3 levels in blood and pleural fluid at presentation in patients with MPM

\section{Outcome measures}

Serum SOMAscan

Plasma fibulin-3

Final diagnosis reached
Serum SOMAscan and plasma fibulin-3 at presentation

Survival (from registration)

Serum SOMAscan and plasma fibulin-3 3 months post-Dx

Survival (from registration)

\section{Serum SOMAscan}

Plasma fibulin-3

MPM tumour volume at MRI, defined using Myrian Intrasense software

Serum SOMAscan

Plasma fibulin-3

The following MRI biomarkers:

- MRI-ECE

- Redistribution rate constant $\left(\mathrm{K}_{\mathrm{ep}}\right)$

- Elimination rate constant $\left(\mathrm{K}_{\mathrm{el}}\right)$

SOMAscan and fibulin-3 at presentation and at 1 month postbiopsy \pm drainage and pleurodesis

DIAPHRAGM, Diagnostic and Prognostic Biomarkers in the Rational Assessment of Mesothelioma; MPM, malignant pleural mesothelioma.

supplementary appendix 2) at clinic. All participants will be invited to a single research clinic visit assuming the following eligibility criteria are met.

Inclusion criteria:

- Documented history of asbestos exposure and associated pleural plaques, asbestosis or diffuse pleural thickening;

- Willing and able to travel to a research clinic interview in Glasgow;

- Informed written consent.

Exclusion criteria

- Known MPM;

- Known or suspected other thoracic malignancy under investigation;

- Known pleural effusion of any cause.

Detailed asbestos exposure histories will be taken from all participants in the SPM cohort and the AEC cohort. This will be done using an asbestos exposure questionnaire derived from the Health and Safety Executive asbestos survey ${ }^{40}$ (see online supplementary appendix $3)$. This questionnaire includes recording of the nature of occupational exposure(s), which can be correlated to likely fibre exposure. The duration and first year of exposure is also recorded. Non-occupational sources of exposure are also recorded (eg, the washing of an occupationally exposed spouse's work clothes). Only AECs with documented imaging sequelae of asbestos exposure (eg, pleural plaques) and an asbestos exposure history will be included.

\section{Cross-sectional MRI substudy}

Fifty patients will be recruited to address the study's exploratory objectives (see table 1). Eligibility will be determined based on the following criteria.

Inclusion criteria:

- Pleural histological sampling (by LAT/image-guided biopsy) indicated to investigate SPM following a nondiagnostic pleural aspiration;

- Recruited in a West of Scotland (WoS) centre.

Exclusion criteria:

- Unable to undergo MRI (claustrophobia or known contraindications such as pacemaker, ferrous metal implants or foreign body);

- Allergy to gadolinium contrast;

- Renal impairment (estimated glomerular filtration rate $<30 \mathrm{~mL} / \mathrm{min}$ );

- Pregnancy.

Based on previous audit data from the host centre, we expect at least $40 \%(\mathrm{n}=20)$ of patients in the substudy to have MPM. Eligible participants will be approached at the clinical visit during which non-diagnostic pleural aspiration results, and the need for further investigation, 
are discussed. Participants will be provided with a separate PIS (see online supplementary appendix 4) and will be asked to provide additional informed written consent.

\section{Consent}

All participants will be given sufficient time (as judged by themselves) to provide written informed consent after reading the relevant PIS and having the opportunity to ask questions.

\section{Outcome measures}

The outcome measures associated with each of the trial's objectives are detailed in table 1.

\section{Final diagnosis}

A specific cytological or histological pleural diagnosis will be sought in all patients according to national guidelines. $^{20}$ This will be recorded as the final diagnosis, which may be based on immediate repeat biopsies felt to be indicated by the site principal investigator (see figure 1). Any cytologically or histologically confirmed non-MPM diagnosis (eg, pleural metastases from lung cancer) will be recorded without the need for any further updates. However, sites will need to provide updates for any non-MPM diagnosis that is not cytologically or histologically confirmed (eg, parapneumonic effusion). These will be submitted on the 12-month anniversary of the original diagnosis, or as soon as any new pleural diagnosis is made. This aims to capture any false-negative diagnostic tests from the initial presentation, acknowledging the major diagnostic challenges posed by pleural malignancies, particularly MPM.

\section{Biomarker sampling and storage}

Blood samples ( \pm pleural fluid in WoS centres) will be drawn and immediate processing performed at each study centre. Samples can be taken before or after pleural aspiration. Patients with positive pleural cytology cannot be recruited (see figure 1A). Duplicate samples will be collected for all measurements at all visits, ensuring redundancy in case of loss or damage to samples during transportation to the appropriate central laboratory. SOMAmer biomarker levels will be measured in serum; therefore, $9 \mathrm{~mL}$ of venous blood will be collected first into a vacutainer tube containing serum separator tube clot activator. Fibulin-3 levels will be measured in plasma; therefore, $9 \mathrm{~mL}$ of venous blood will be collected second into a vacutainer tube containing EDTA. In centres contributing to the exploratory MRI substudy (WoS sties only) $20 \mathrm{~mL}$ of pleural fluid will be also collected into a plain container if pleural fluid is being drawn for diagnostic/therapeutic purposes at the same visit. If not done at this first opportunity, prediagnosis pleural fluid can also be collected during local anaesthetic or general anaesthetic thoracoscopy, prior to any biopsy or pleurodesis being performed.
Biomarker processing and storage

Serum samples will be allowed to clot for 30 min before centrifugation. Plasma and pleural fluid samples will be centrifuged immediately. All samples will be centrifuged at $2200 \mathrm{~g}$ for $15 \mathrm{~min}$ at room temperature. For all samples, the supernatant will be withdrawn by pipette, aliquoted into cryovials of at least $250 \mu \mathrm{L}$ volume, labelled and placed into a $-80^{\circ} \mathrm{C}$ freezer within 2 hours. Samples will be stored at each recruiting centre until batched transport to the appropriate study laboratory. Samples from WoS recruiting centres will be used to create a bioresource. The bioresource will be stored as a satellite collection of the NHS Greater Glasgow and Clyde Biorepository, a Health Improvement Scotland (HIS)-approved tissue bank. Data will be stored in the secure Cancer Research UK (CRUK) Clinical Trials Unit (CTU) database. On study completion, investigators will be invited to apply for access to data and samples appropriate to their research questions. This will allow external validation of new markers, including those reported since the study's design (such as High Mobility Group Box-1 (HMGB-1)), ${ }^{41}$ in an intention-to-diagnose population. Access will be granted after peer review of each proposal by a scientific board comprising members of the DIAPHRAGM Trial Management Group (TMG) and senior biorepository staff. An annual update on this activity will be submitted to the WoS Research Ethics Committee.

\section{Biomarker analyses}

SomaLogic (Boulder, Colorado, USA) will perform all SOMAscan proteomic analyses. ${ }^{27}$ This uses SOMAmer reagents to specifically bind to protein targets in blood. Relative protein concentrations will be converted to measurable nucleic acid signals that are quantified by hybridisation to DNA microarrays. ${ }^{28}$

Fibulin-3 and mesothelin levels will be measured by the Translational Pharmacology Unit, Wolfson Wohl Cancer Research Centre, UK, using ELISA methods validated according to the Food and Drug Administration (FDA)-recommended guidelines for bioanalytical methods. ${ }^{42}$ Fibulin-3 levels in plasma and pleural fluid will be measured using the commercially available ELISA (Cloud-Clone Corp, formerly USCN Life Science, Houston, Texas, USA) as in the original Pass et al $\mathrm{s}^{25}$ study. Mesothelin will be measured using the MESOMARK ELISA (Fujirebio Diagnostics).

\section{MRI}

Patients will be scanned at the Queen Elizabeth University Hospital, Glasgow, on a 3.0T Siemens Verio MRI Scanner. After localisation of the affected thoracic cavity, an isotropic three-dimensional T1-weighted volume will be acquired using volumetric interpolated breath-hold examination (VIBE) sequences. A stack of axial slices covering the entire lung and surrounding pleura will be acquired as a set of short breath holds. Gadolinium-diethylenetriamine pentaacetic acid contrast 
(Gadovist) will be administered via a peripheral intravenous line as a $15-40 \mathrm{~mL}$ bolus $(0.05 \mathrm{mmol} / \mathrm{kg})$. VIBE sequences will be reacquired at copied slice positions to provide precontrast and postcontrast images. The total scan time will be around $45 \mathrm{~min}$. Regions of enhancing pleural tumour will be defined using semiautomated signal intensity thresholding based on contrast-enhanced axial slices using Myrian Intrasense software, which has previously been used to assess tumour volume in MPM. ${ }^{43}$ MRI volumetry analyses will be validated using imaging phantoms.

\section{Survival}

Survival will be recorded in days from the date of study registration to the data of death, from any cause.

\section{Sample size, assumptions and uncertainties}

Sample size estimations for each marker were based on published data at the point of study design and a projected MPM incidence of 13-20\% in the SPM cohort. The power available to test the hypotheses below is therefore reported as a range, based on final MPM numbers lying between 83 (13\% incidence) and 120 (20\% incidence).

\section{Primary objective \\ SOMAscan assay}

We hypothesise that the MPM sensitivity and specificity exceed $90 \%$, based on previously reported performance in combined training, verification and validation sets (sensitivity $93.2 \%$ ( $88.6 \%$ to $97.7 \%$ ), specificity (90.8\% $\left.(86.1 \% \text { to } 95.6 \%)^{27}\right)$. Recruitment of $83-120$ patients with MPM will allow us to distinguish a sensitivity of $>90 \%$ from a sensitivity $<80 \%$ with $80-93 \%$ power, respectively, at the $5 \%$ one-sided level of significance. In total, 83-120 patients with MPM will allow discrimination between a specificity $<80 \%$ and a specificity $>90 \%$, with $80-88 \%$ power at the $5 \%$ one-sided level of statistical significance. The SE in the estimated sensitivity and specificity will be $<5 \%$, across all possible outcomes.

\section{Fibulin-3}

We hypothesise that the MPM sensitivity will exceed $80 \%$ and that the specificity will exceed $90 \%$ (at the $52 \mathrm{ng} /$ $\mathrm{mL}$ cut-off). These figures are based on a reduced level of performance to the primary results reported by Pass et $a l^{25}$ (97\% sensitivity, 95\% specificity), given lower sensitivity in the external validation cohort studied $(40 \%$ at $95 \%$ specificity).

With 83-120 patients with MPM, the study will be able to distinguish a sensitivity of $>80 \%$ from a sensitivity $<70 \%$ with $65-80 \%$ power, respectively, at the $5 \%$ onesided level of statistical significance. The SE in the estimated sensitivity will be $<5 \%$. In order to achieve $90 \%$ power to distinguish a specificity of $>90 \%$ from a specificity $<85 \%$ at the $5 \%$ one-sided level of statistical significance, a random sample of 378 non-MPM samples will be analysed. The SE in the estimated specificity will be $<2.3 \%$.

The study data will be used to estimate the AUC for the SOMAscan marker for distinguishing patients with MPM from non-MPM patients in the SPM cohort. Assuming 83-120 patients in the MPM group and 83120 in the non-MPM group, the AUC can be estimated with a $95 \%$ CI of width 0.120 to 0.168 (assuming a cutpoint exists with a reasonable sensitivity of $80 \%$ and a modest specificity of $40 \%$ ). If more sensitive/specific cut-points exist, the width of the $95 \%$ CI will be much reduced. The study data will be used to develop a new diagnostic signature based on fibulin-3 and SOMAscan results to distinguish MPM from non-MPM effusions.

\section{Secondary objectives}

The study data will be used to determine whether baseline SOMAscan results and/or fibulin-3 levels, or a change in levels at 3 months (fibulin-3 only), are independent prognostic factors for MPM. A correlation of 0.4 between existing prognostic factors and each marker has been assumed. For the baseline levels, to detect an approximate doubling in median overall survival (from 6 to 12 months-a HR of 2) with $80 \%$ power and $5 \%$ twosided level of statistical significance between a good/poor prognostic group based on dichotomising these markers requires at least 83 patients with MPM recruited over 3 years with $\sim 6$ months subsequent follow-up to observe 66 deaths. For the 3-month change levels, a HR of 2.38 can be detected ( $80 \%$ power, $5 \%$ two-sided level of statistical significance) when 49 deaths are observed in the estimated 66 out of 83 patients who survive to 3 months.

\section{Exploratory objectives}

These will be addressed in the MRI substudy, which will generate a sample of at least 20 patients with MPM. This will allow moderately large associations (0.6) between the exploratory outcome measures (see table 1) to be detected at $80 \%$ power at the $5 \%$, two-sided level of statistical significance. The effect of pleural biopsies \pm drainage/pleurodesis on fibulin-3 levels will be assessed using all 50 patients recruited. This will allow moderately small differences (standardised difference of 0.4 ) to be detected with $80 \%$ power at the $5 \%$ two-sided level of statistical significance.

\section{Statistical analysis plan}

\section{Primary analysis}

Sensitivity and specificity at prespecified cut-offs will be estimated using standard approaches for proportions. The diagnostic performance of each biomarker will be assessed using ROC curves. All patients with MPM ( $n=83-120)$ will be included and compared with AECs and a random sample of non-MPM cases. Owing to cost constraints related to SOMAscan analyses, 83 AECs and 83 non-MPM cases will be randomly selected. All AECs and 378 non-MPM cases will be used for fibullin-3 analyses and for comparison with mesothelin. Logistic 
regression will be used to estimate a diagnostic model using biomarker results and clinical or radiological variables. Cross-validation will be used to provide robust estimates of AUC and specificity at fixed sensitivity rates of $80 \%, 90 \%$ and $95 \%$.

\section{Secondary analysis}

A prognostic model will be developed using Cox proportional hazard techniques. The modelling process will incorporate biomarker measurements (at presentation (both markers) and at 3 months (fibulin-3 only) and other known prognostic features (eg, performance status, histology).

\section{Exploratory analysis}

The association between SOMAscan results/fibulin-3 in blood and tumour volume/measures of tumour angiogenesis will be estimated by Pearson or Spearman correlation, depending on the normality of the data. The same methods will be used to test the association between fibulin-3 in blood and pleural fluid. Changes in fibulin-3 levels before and after histological sampling (at 1 month follow-up) will be compared using a paired t-test or Wilcoxon signed rank-sum test (depending on the normality of the data). Owing to cost constraints, exploratory end points involving pleural fluid SOMAscan results will be analysed at a later date.

\section{Changes to the study protocol since trial opening}

The protocol described accurately reflects V.5, of the protocol, dated 17/6/16. The following changes were made in previous versions:

- V.2, dated 14/2/14:

- Safety reporting reduced following risk assessment by study sponsor.

- Collection of duplicate blood samples as provision for loss or damage and for sample retention in tissue bank.

- Greater flexibility to timing of first blood draw.

- V.3, dated 17/10/14:

- Addition of recruitment of controls from respiratory medicine clinics.

- Addition of exclusion criteria for patients with chest drains in situ.

- Eligibility for the MRI substudy extended to patients proceeding to image-guided pleural biopsy.

- V.4, dated 27/4/15:

- Update to the exclusion criteria for the AECs to include known or suspected thoracic malignancy under investigation.

- V.5, dated 17/6/16:

- Power projections adjusted based on interim reporting of MPM incidence from recruiting centres.

\section{Definition of end of study}

The trial will end 2 years after the last patient with confirmed MPM is recruited or whenever all patients with MPM have died (whichever occurs first).
Monitoring, data management and quality assurance

No on-site monitoring will be undertaken. Two telephone-monitoring calls will be conducted by a CRUK Glasgow CTU Monitor to carry out process, compliance and documentation checks. Central monitoring of trial data will be performed by the trial statistician and clinical trial coordinator by checking incoming forms for compliance with the protocol, data consistency, missing data and timing. The CRUK Glasgow CTU will control data consistency and data quality by entering trial data onto CTU database. Computerised and manual consistency checks will be performed and queries issued in cases of inconsistency or missing information. An audit trail of changes to the database will be maintained.

\section{Safety considerations}

Participants in the MRI substudy will be asked at their 1-month follow-up visit about the occurrence of adverse events related to the administration of MRI contrast (gadolinium). These will be followed until resolution.

\section{Dissemination}

The results of the study will be presented at national and international scientific meetings and published in full in a peer-reviewed journal (authorship will be according to that journal's guidelines). A lay summary will be produced and disseminated to interested parties.

\section{Trial management}

The trial will be coordinated from the CRUK Glasgow CTU by the TMG, including the chief investigator, selected co-investigators, project manager, trial statistician, clinical trial coordinator and IT staff. The TMG will oversee the running of the trial and meet monthly.

\section{Author affiliations}

${ }^{1}$ Department of Respiratory Medicine, Queen Elizabeth University Hospital, Glasgow, UK

${ }^{2}$ Institute of Cancer Sciences, University of Glasgow, Glasgow, UK

${ }^{3}$ Cancer Research UK Glasgow Clinical Trials Unit, UK

${ }^{4}$ Glasgow Clinical Research Imaging Facility, Queen Elizabeth University Hospital, Glasgow, UK

${ }^{5}$ Department of Radiology, Queen Elizabeth University Hospital, Glasgow, UK ${ }^{6}$ Academic Respiratory Unit, School of Clinical Sciences, University of Bristol, Bristol, UK

${ }^{7}$ Beatson West of Scotland Cancer Centre, Glasgow, UK

${ }^{8}$ Institute of Infection, Immunology and Inflammation, University of Glasgow, Glasgow, UK

Acknowledgements The authors are grateful to the Chief Scientist Office (Scotland) as the study funder, cancer research networks infrastructure, the participating sites and staff, SomaLogic Clydeside Action on Asbestos and the patients involved. ST is part funded by the West of Scotland Lung Cancer Research Fund. KGB is part funded by a NHS Career Research Fellowship. CRUK and ECMC are acknowledged by FT and CM.

Contributors ST and JEF contributed to the conception or design of the work; data acquisition, analysis and interpretation of data for the work. CK contributed to the design of the work; analysis and interpretation of data for the work. LA and DBS contributed to the design of the work and interpretation of data for the work. CM, FT and RW contributed to the design 
of the work; data acquisition, analysis or interpretation of data for the work. JP contributed to the conception and design of the work; data analysis or interpretation of data for the work. NAM contributed to the design of the work; data analysis and interpretation of data for the work. AC contributed to the conception and design of the work; interpretation of data for the work. ST, CK, LA, CM, FT, RW, JEF, DBS, JP, NAM and AC were involved in revising the work critically for important intellectual content, final approval of the version to be published, and agree to be accountable for all aspects of the work in ensuring that questions related to the accuracy or integrity of any part of the work are appropriately investigated and resolved. KGB provided principal contribution to the conception and design of the work; data acquisition, analysis and interpretation of data for the work; drafting the work; final approval of the version to be published; and agrees to be accountable for all aspects of the work in ensuring that questions related to the accuracy or integrity of any part of the work are appropriately investigated and resolved.

Funding This work was supported by the Chief Scientist's Office of the Scottish Government (Project Grant ETM/285) and the West of Scotland Lung Cancer Research Group (Award September 2015). KGB is part-funded by NHS Research Scotland.

Competing interests SomaLogic have provided funding for all SOMAscan assays.

Ethics approval The study protocol, all documents and amendments have been approved by the West of Scotland Research Ethics Service (Ref: 13/WS/ 0240).

Provenance and peer review Not commissioned; externally peer reviewed.

Open Access This is an Open Access article distributed in accordance with the Creative Commons Attribution Non Commercial (CC BY-NC 4.0) license, which permits others to distribute, remix, adapt, build upon this work noncommercially, and license their derivative works on different terms, provided the original work is properly cited and the use is non-commercial. See: http:// creativecommons.org/licenses/by-nc/4.0/

\section{REFERENCES}

1. Musk AW, Olsen $\mathrm{N}$, Alfonso $\mathrm{H}$, et al. Predicting survival in malignan mesothelioma. Eur Respir J 2011;38:1420-4.

2. Beckett $P$, Edwards J, Fennell D, et al. Demographics, management and survival of patients with malignant pleural mesothelioma in the National Lung Cancer Audit in England and Wales. Lung Cancer 2015:88:344-8.

3. Tsim S, Dick C, Roberts F, et al. 76 Early experience of a regional mesothelioma MDT in the West of Scotland. Lung Cancer 2014;83 (Suppl 1):S28-9.

4. Renshaw AA, Dean BR, Antman $\mathrm{KH}$, et al. The role of cytologic evaluation of pleural fluid in the diagnosis of malignant mesothelioma. Chest 1997;111:106-9.

5. Scherpereel A, Astoul P, Baas P, et al. Guidelines of the European Respiratory Society and the European Society of Thoracic Surgeons for the management of malignant pleural mesothelioma. Eur Respir J 2010;35:479-95.

6. Rusch VW, Giroux D. Do we need a revised staging system for malignant pleural mesothelioma? Analysis of the IASLC database. Ann Cardiothorac Surg 2012;1:438-48.

7. Boutin C, Rey F. Thoracoscopy in pleural malignant mesothelioma: a prospective study of 188 consecutive patients. Part 1: diagnosis. Cancer 1993;72:389-93.

8. Salyer WR, Eggleston JC, Erozan YS. Efficacy of pleural needle biopsy and pleural fluid cytopathology in the diagnosis of malignant neoplasm involving the pleura. Chest 1975;67:536-9.

9. Prakash UB, Reiman HM. Comparison of needle biopsy with cytologic analysis for the evaluation of pleural effusion: analysis of 414 cases. Mayo Clin Proc 1985;60:158-64.

10. Nance KV, Shermer RW, Askin FB. Diagnostic efficacy of pleural biopsy as compared with that of pleural fluid examination. Mod Pathol 1991;4:320-4.

11. O'Rourke N, Garcia JC, Paul J, et al. A randomised controlled trial of intervention site radiotherapy in malignant pleural mesothelioma. Radiother Oncol 2007;84:18-22.

12. Boutin C, Rey F, Viallat JR. Prevention of malignant seeding after invasive diagnostic procedures in patients with pleural mesothelioma. A randomized trial of local radiotherapy. Chest 1995;108:754-8.

13. Robinson BW, Creaney J, Lake R, et al. Mesothelin-family proteins and diagnosis of mesothelioma. Lancet 2003;362: 1612-16.

14. Creaney J, Dick IM, Meniawy TM, et al. Comparison of fibulin-3 and mesothelin as markers in malignant mesothelioma. Thorax 2014;69:895-902

15. Creaney J, Yeoman D, Demelker Y, et al. Comparison of osteopontin, megakaryocyte potentiating factor, and mesothelin proteins as markers in the serum of patients with malignant mesothelioma. J Thorac Oncol 2008;3:851-7.

16. Pass HI, Lott D, Lonardo F, et al. Asbestos exposure, pleural mesothelioma, and serum osteopontin levels. $N$ Engl J Med 2005;353:1564-73.

17. Chang K, Pai LH, Batra JK, et al. Characterization of the antigen (CAK1) recognized by monoclonal antibody $\mathrm{K} 1$ present on ovarian cancers and normal mesothelium. Cancer Res 1992;52. 181-6.

18. Chang $\mathrm{K}$, Pai $\mathrm{LH}$, Pass $\mathrm{H}$, et al. Monoclonal antibody $\mathrm{K} 1$ reacts with epithelial mesothelioma but not with lung adenocarcinoma. Am J Surg Pathol 1992;16:259-68.

19. Hollevoet K, Nackaerts K, Thimpont J, et al. Diagnostic performance of soluble mesothelin and megakaryocyte potentiating factor in mesothelioma. Am J Respir Crit Care Med 2010;181: 620-5.

20. Hooper C, Lee YCG, Maskell N, et al. Investigation of a unilateral pleural effusion in adults: British Thoracic Society Pleural Disease Guideline 2010. Thorax 2010;65(Suppl 2):ii4-17.

21. Hollevoet K, Nackaerts K, Thas O, et al. The effect of clinical covariates on the diagnostic and prognostic value of soluble mesothelin and megakaryocyte potentiating factor. Chest 2012;141:477-84

22. Bergamini S, Bellei E, Bonetti LR, et al. Inflammation: an important parameter in the search of prostate cancer biomarkers. Proteome Sci 2014;12:32.

23. Yuan JJ, Coplen DE, Petros JA, et al. Effects of rectal examination, prostatic massage, ultrasonography and needle biopsy on serum prostate specific antigen levels. J Urol 1992;147:810-14.

24. Wong V, Wang DY, Warren $\mathrm{K}$, et al. The effects of timing of fine needle aspiration biopsies on gene expression profiles in breast cancers. BMC Cancer 2008;8:277.

25. Pass HI, Levin SM, Harbut MR, et al. Fibulin-3 as a blood and effusion biomarker for pleural mesothelioma. $N$ Engl J Med 2012;367:1417-27.

26. Kirschner MB, Pulford E, Hoda MA, et al. Fibulin-3 levels in malignant pleural mesothelioma are associated with prognosis but not diagnosis. Br J Cancer 2015;113:963-9.

27. Ostroff RM, Mehan MR, Stewart A, et al. Early detection of malignant pleural mesothelioma in asbestos-exposed individuals with a noninvasive proteomics-based surveillance tool. PLOS ONE 2012;7:e46091.

28. Gold L, Ayers D, Bertino J, et al. Aptamer-based multiplexed proteomic technology for biomarker discovery. PLOS ONE 2010;5: e15004.

29. Kao SCH, Pavlakis N, Harvie R, et al. High blood neutrophil-to-lymphocyte ratio is an indicator of poor prognosis in malignant mesothelioma patients undergoing systemic therapy. Clin Cancer Res 2010;16:5805-13.

30. Hooper CE, Lyburn ID, Searle J, et al. The South West Area Mesothelioma and Pemetrexed trial: a multicentre prospective observational study evaluating novel markers of chemotherapy response and prognostication. Br J Cancer 2015;112:1175-82.

31. Pinato DJ, Mauri FA, Ramakrishnan R, et al. Inflammation-based prognostic indices in malignant pleural mesothelioma. J Thorac Oncol 2012;7:587-94.

32. Burt BM, Rodig SJ, Tilleman TR, et al. Circulating and tumor-infiltrating myeloid cells predict survival in human pleura mesothelioma. Cancer 2011;117:5234-44.

33. Zhang Y, Marmorstein LY. Focus on molecules: fibulin-3 (EFEMP1). Exp Eye Res 2010;90:374-5

34. Agha MA, El-Habashy MM, El-Shazly RA. Role of fibulin-3 in the diagnosis of malignant mesothelioma. Egypt $J$ Chest Dis Tuberc 2014;63:99-105.

35. Corradi M, Goldoni M, Alinovi R, et al. YKL-40 and mesothelin in the blood of patients with malignant mesothelioma, lung cancer and asbestosis. Anticancer Res 2013;33:5517-24.

36. Creaney J, Francis RJ, Dick IM, et al. Serum soluble mesothelin concentrations in malignant pleural mesothelioma: relationship to tumor volume, clinical stage and changes in tumor burden. Clin Cancer Res 2011;17:1181-9. 
37. Pairon JC, Laurent F, Rinaldo $\mathrm{M}$, et al. Pleural plaques and the risk of pleural mesothelioma. J Natl Cancer Inst 2013;105: 293-301.

38. Paris C, Thierry S, Brochard P, et al. Pleural plaques and asbestosis: dose- and time-response relationships based on HRCT data. Eur Respir J 2009;34:72-9.

39. Baldwin DR, Callister MEJ, Guideline Development Group. The British Thoracic Society guidelines on the investigation and management of pulmonary nodules. Thorax 2015;70:794-8.

40. HSE. The Asbestos Survey. 2016:1-150. http://www.hse.gov.uk
41. Napolitano A, Antoine DJ, Pellegrini L, et al. HMGB1 and its hyperacetylated isoform are sensitive and specific serum biomarkers to detect asbestos exposure and to identify mesothelioma patients. Clin Cancer Res 2016;22:3087-96.

42. Services UDOHAH. Guidance for industry bioanalytical method validation. 2001. http://www. fda. gov/downloads/Drugs/ GuidanceComplianceRegulator y Information

43. Frauenfelder T, Tutic M, Weder W, et al. Volumetry: an alternative to assess therapy response for malignant pleural mesothelioma? Eur Respir J 2011;38:162-8. 